\title{
Evaluation of various common prostate IMRT techniques based on estimated tumor control and normal tissue complication probabilities in correlation with patients anatomical parameters derived from the CT scans
}

\author{
Amin BANAEI ${ }^{1}$, Bijan HASHEMI ${ }^{1, a}$, Mohsen BAKHSHANDEH ${ }^{2}$, Bahram MOFID ${ }^{3}$ \\ ${ }^{1}$ Department of Medical Physics, Faculty of Medical Sciences, Tarbiat Modares University, Tehran, Iran \\ ${ }^{2}$ Department of Radiology Technology, Faculty of Allied Medical Sciences, Shahid Beheshti University of Medical Sciences, \\ Tehran, Iran \\ ${ }^{3}$ Department of Radiation Oncology, Faculty of Medical Sciences, Shahid Beheshti University of Medical Sciences, \\ Tehran, Iran \\ ${ }^{a}$ E-mail address: bhashemi@modares.ac.ir
}

(received 21 July 2018; revised 9 December 2018; accepted 15 January 2019)

\begin{abstract}
Background: The relationship between the prostate IMRT techniques and patients anatomical parameters has been rarely investigated.

Objective: to evaluate various prostate IMRT techniques based on tumor control and normal tissue complication probability (TCP and NTCP) values and also the correlation of such techniques with patients anatomical parameters.

Methods: Four IMRT techniques (9, 7 and 5 fields and also automatic) were planned on the CT scans of 63 prostate cancer patients. The sum of distances between the organs at risk (OARs) and target tissue and also their average joint volumes were measured and assumed as anatomical parameters. Selected dosimetric and radiobiological parameters (TCP and NTCP) values were compared among various techniques and the correlation with the above anatomical parameters were assessed using Pearsons' correlation.

Results: High correlations were found between the dosimetric/radiobiological parameters of OARs with the joint volumes and with the distances between the OARs and target tissue in all the techniques. The TCP and complication free tumor control probability $\left(\mathrm{P}_{+}\right)$values were decreased with increasing the joint volume and decreasing the distances between the OARs and target tissue (as poly-nominal functions). The NTCP values were increased with increasing the joint volumes and decreasing the distances (3-degree poly-nominal functions). For the low percent joint volumes $(<20 \%)$ and high distances $(>7 \mathrm{~cm})$, The TCP, NTCP and $\mathrm{P}_{+}$showed no statistical differences between various techniques (P-value $>0.07)$. However, 9 and 7 fields techniques indicated better radiobiological results $(\mathrm{P}$-value $<0.05)$ in almost other ranges ( $>20 \%$ joint volumes and $<7 \mathrm{~cm}$ distances).

Conclusion: Based on our results, it would be possible to compare radiobiological effects of various common IMRT techniques and choose the best one regarding to patients anatomical parameters derived from the CT scans.
\end{abstract}

Key words: prostate cancer; intensity-modulated radiotherapy; radiobiology; TCP; NTCP; anatomical parameters.

\section{Background}

Prostate cancer is one of the most common cancers among men [1]. Radiotherapy as one of the treatment methods has a special place in the treatment of this cancer at various stages. Radiotherapy is used for the patients at various stages with different doses and therapeutic goals [2]. Intensity modulated radiotherapy (IMRT), as a one of most usable techniques [3], is used to deliver prescribed dose to the target tissue and spare OARs from irradiation. The preference of IMRT on conventional and conformal techniques has been proven in previous studies [4-7]. In addition, IMRT has indicated similar or just a little worse radiobiological and dosimetric results in comparison with newer radiotherapy techniques, like the volumetric modulated radiotherapy, tomotherapy and intensity modulated arc therapy [8-10].

Although the preference of IMRT techniques on conventional and conformal techniques has been reported in previous studies, comparison at various IMRT techniques has not adequately been investigated especially based on radiobiological factors. There are several studies investigating the effect of beam orientation and numbers in IMRT [11-13]. However, in such studies, usually an algorithm or software with the ability of beam angle optimization has been used or proposed. Furthermore, these studies have not compared different available or common techniques. This may be due to 
various anatomical specifications of patients. The role of anatomical parameters for the assessment of IMRT treatment planning has also rarely been investigated $[14,15]$.

Several anatomical parameters can be measured through the CT and MRI scans of patients. The volumes of prostate, planning target volume (PTV), OARs, joint volumes between the PTV with OARs, distances between the femur heads and distances between the OARs and target, can be regarded as such parameters. Investigating the correlation between such parameters and the IMRT dosimetric/radiobiological results may be useful in comparing various common IMRT techniques and choosing the best appropriate one meeting the treatment goals. However, most of the anatomical parameters by themselves, cannot determine the differences between the IMRT techniques, because they just reflect the specifications of either one of the OARs or the target tissue. To achieve the radiotherapy goal, there is a need for the parameters that account both of the OARs and target tissue specifications. Therefore, the joint volumes and distances between the OARs and target tissue were the anatomical parameters candidates we chose for evaluating various IMRT techniques in different patients. The joint volumes must receive the target prescribed dose. On the other hand, such volumes must be spared from any irradiation. Therefore, variation of this anatomical parameter may have high influence on dosimetric and radiobiological results of IMRT planning. In addition, increasing the distance between the centers of OARs and target tissue helps us to prevent OARs from high dose regions and also reduce the joint volume of PTV and OARs. Assessment the effects of dosimetric and radiobiological results of various IMRT techniques in correlation with these parameters has not previously been investigated.

We aimed to evaluate and find the radiobiological effects of various IMRT techniques, including 9, 7 and, 5 fields as well as automatic beam angle determination technique on the target and OARs (rectum and bladder), using the Relative Seriality model [16] for prostate cancer patients with various anatomical parameters of our interest. In other word, finding the relationship between such anatomical parameters and radiobiological results of various prostate IMRT techniques was the goal of our study.

\section{Methods}

The CT (Matrix size: 512 x 512; Slice thickness: 3 to $5 \mathrm{~mm}$ ) and T2w-MRI (1.5 Tesla with fast spin echo pulse sequence with TE: $100 \mathrm{~ms}$ and TR: $3000 \mathrm{~ms}$ ) images were taken from 63 patients with prostate cancer at $\mathrm{T} 2 \mathrm{a}$ to $\mathrm{T} 3 \mathrm{~b}$ stages. All the patients were instructed ahead to have an empty rectum along with the comfortably full bladder and were placed in supine position for both of the imaging procedures. The MRI and CT images of every patient were merged to determine precisely the radiation-sensitive organs based on their bony landmarks.

The patients' radiotherapy plans were designed with Eclipse software (version 11, manufactured by Varian Corporation,
US). The gross tumor volume was the same as the clinical tumor volume, including prostate and seminal vesicles. A margin of $1 \mathrm{~cm}$ thickness was chosen around the tumor volume in all directions, except the posterior which was $0.8 \mathrm{~cm}$ indicating the PTV. The prescription was to deliver a total dose of $70.2 \mathrm{~Gy}$ in 26 fractions (hypofractionated radiotherapy) to the PTV uniformly and prevent the dose reached to surrounding normal structures at the lowest possible level. Four IMRT methods including 9-fields (briefly 9F with gantry angles of $0,30,60,105,140,220,270,300,330$ ), 7-fields (briefly $7 \mathrm{~F}$ with gantry angles of $0,50,110,155,205,250$, 310 ) and 5-fields (briefly 5F with gantry angles of $0,75,135$, $225,285)$ and also the automatic beam selection methods (beam angle optimization or briefly BAO) were used. The Number and angle of the fields in the BAO method, were determined by the treatment planning software. During the course of radiation therapy, the radiation field size was adjusted to cover the target by multileaf collimators (MLC) system. The MLC system was a Varian's 80L model, containing 40 pairs of lead leaves.

All plans were interactively optimized based on our institutional planning protocol derived from a previous study by Pollak et al. [17] in which more than or equal to $98 \%$ of PTV volume received $70.2 \mathrm{~Gy}$ and no more than $2 \%$ of the PTV received 75 Gy or higher doses. Furthermore, the volumes of bladder and rectum that received $50 \mathrm{~Gy}$ or lower were chosen in a way to be less than $25 \%$ and $17 \%$ respectively. In addition, the volumes of bladder and rectum that received 31 Gy or lower were less than $50 \%$ and $35 \%$ respectively and the maximum dose of $40 \mathrm{~Gy}$ was considered for femur heads.

The dose volume histograms (DVHs) of OARs and CTV for the IMRT plans of each patient were entered in the BioSuite software [18]. The TCP and NTCP were calculated for the CTV and OARs respectively using the relative seriality model [16] using radiobiological model parameters from the study by Deb and Fielding [6]. The $\mathrm{P}_{+}$was calculated from the TCP and overall NTCP.

In addition to the radiobiological parameters, the dosimetric parameters including the homogeneity index (HI) for the target tissue calculated based on the ICRU (International Commission on Radiation Units) report 83 [19], the mean dose (Dmean), the volume that received a minimum dose of $50 \mathrm{~Gy}$ (V50) and the volume which received a minimum dose of $60 \mathrm{~Gy}$ (V60) for both of the bladder and rectum were calculated in our study.

The joint volumes percentage and distances (center to center) between OARs with target tissue were considered as the patients anatomical parameters. The Pearsons correlation coefficients were calculated between the OARs dosimetric/ radiobiological parameters with anatomical parameters.

The sum of distances between the center of prostate and rectum/bladder and also the average percentage of joint volumes between the PTV and bladder/rectum were calculated for each patient. Radiobiological results were compared among various IMRT techniques, for different ranges of the anatomical parameters using repeated measurement statistical test. 


\section{Results}

Table 1 shows the means and standard deviations of the dosimetric and radiobiological parameters obtained for the patients. Table 2 shows the calculated Pearsons correlation coefficients between the anatomical parameters with the OARs dosimetric and radiobiological parameters for various IMRT techniques. The OARs radiobiological/dosimetric parameters have high to very high inverse correlations with the distances between the OAR and prostate central positions. High direct correlations were also noted between the OARs radiobiological/dosimetric parameters with the joint volume percentage of the OARs and PTV. Figures 1 and 2 show the relationship between the anatomical parameters with the NTCP of OARs and overall NTCP in all the patients for all the IMRT techniques. In which, vertical lines represent the ranges of anatomical parameters.

Regarding the rectum joint volume with PTV, for the patients with joint volumes ranged from 0 to 20 percent, the 5 fields technique indicated significant higher NTCP values $(\mathrm{P}-$ value $<0.04)$. In higher ranges, both of the 5 fields and $\mathrm{BAO}$ techniques showed higher NTCP values (P-value<0.05). The 9 and 7 fields did not show any statistical differences (pvalue $>0.1$ ) in all of the ranges (Figure 1a).

Regarding to the patients bladder joint volumes with PTV, in the ranges of 0 to $40 \%$, there was no statistical differences (Pvalue>0.06) between the NTCP values of various IMRT techniques (Figure 1b). However, for the patients, with more than $40 \%$ of joint volumes, both of the 9 and 7 fields techniques, resulted significant lower NTCP values (Pvalue<0.03).

Regarding the distances between the OARs and prostate central position, for the distances ranged from 2 to $3 \mathrm{~cm}$ for the rectum (Figure 1c) and 2 to $3.5 \mathrm{~cm}$ for the bladder (Figure 1d), the 9 and 7 fields techniques showed significant lower NTCP values (P-value<0.05). But for higher distances there was no statistical differences between the IMRT techniques (P-value>0.1).

Regarding the average joint volume between the OARs and PTV, there was no significant differences (P-value>0.12) among the overall NTCP of various IMRT techniques for the patients with joint volumes ranged from $5 \%$ to $20 \%$ (Figure 2a). However, for patients with higher average joint volumes, the 9 and 7 fields techniques had significant lower values ( $\mathrm{P}$-value $<0.04)$.

Regarding the sum of the distances between the prostate and OARs central positions, the overall NTCP for both of the 9 and 7 fields technique had lower values $(\mathrm{P}$-value $<0.05)$ in all the ranges (4.9 to $11 \mathrm{~cm}$ ). Furthermore, the BAO techniques showed lower NTCP values $(\mathrm{P}$-value=0.042) in comparison with 5 fields technique (Figure 2b).

Table 1. The means and standard deviations of dosimetric and radiobiological parameters resulted from various IMRT techniques.

\begin{tabular}{|c|c|c|c|c|c|c|c|c|c|c|c|c|}
\hline \multirow{2}{*}{$\begin{array}{c}\text { IMRT } \\
\text { techniques }\end{array}$} & \multicolumn{4}{|c|}{ Bladder } & \multicolumn{4}{|c|}{ Rectum } & \multicolumn{2}{|c|}{ Target Tissue } & \multirow{2}{*}{$\begin{array}{c}\text { Overall } \\
\text { NTCP } \\
(\%)\end{array}$} & \multirow[b]{2}{*}{$\mathbf{P}_{+}(\%)$} \\
\hline & $V_{60}(\mathbf{G y})$ & $\mathrm{V}_{50}(\mathbf{G y})$ & $\begin{array}{c}\text { Mean Dose } \\
\text { (Gy) }\end{array}$ & $\begin{array}{c}\text { NTCP } \\
(\%)\end{array}$ & $V_{60}(\mathbf{G y})$ & $\mathrm{V}_{50}(\mathbf{G y})$ & $\begin{array}{c}\text { Mean Dose } \\
\text { (Gy) }\end{array}$ & $\begin{array}{c}\text { NTCP } \\
(\%)\end{array}$ & HI $^{*}$ & TCP (\%) & & \\
\hline 9 fields & $27.86 \pm 5.38$ & $37.59 \pm 6.72$ & $38.17 \pm 4.21$ & $1.96 \pm 1.77$ & $18.91 \pm 6.27$ & $28.52 \pm 6.28$ & $35.92 \pm 4.77$ & $2.48 \pm 1.97$ & $0.129 \pm 0.024$ & $96.02 \pm 2.44$ & $4.44 \pm 3.12$ & $88.81 \pm 16.71$ \\
\hline 7 fields & $27.91 \pm 5.67$ & $38.09 \pm 6.31$ & $38.22 \pm 4.37$ & $2.01 \pm 1.80$ & $19.47 \pm 7.34$ & $29.51 \pm 7.17$ & $37.89 \pm 4.67$ & $2.51 \pm 1.93$ & $0.138 \pm 0.027$ & $95.96 \pm 2.29$ & $4.51 \pm 3.15$ & $88.67 \pm 16.64$ \\
\hline 5 fields & $27.66 \pm 5.43$ & $36.43 \pm 5.94$ & $39.77 \pm 4.52$ & $2.26 \pm 2.27$ & $20.23 \pm 6.66$ & $29.99 \pm 7.24$ & $35.97 \pm 4.62$ & $2.88 \pm 2.19$ & $0.186 \pm 0.029$ & $95.25 \pm 2.73$ & $5.13 \pm 3.73$ & $87.39 \pm 16.78$ \\
\hline $\mathrm{BAO}^{*}$ & $27.56 \pm 7.07$ & $35.18 \pm 7.15$ & $38.91 \pm 5.49$ & $2.24 \pm 2.67$ & $19.65 \pm 8.21$ & $28.30 \pm 9.48$ & $36.37 \pm 5.71$ & $2.66 \pm 2.17$ & $0.159 \pm 0.039$ & $95.57 \pm 2.79$ & $4.89 \pm 3.99$ & $87.93 \pm 16.99$ \\
\hline
\end{tabular}

Table 2. The calculated Pearson correlation coefficients between the anatomical parameters with the OARs dosimetric (V50, V60, Dmean) and radiobiological (NTCP) parameters for various IMRT techniques.

\begin{tabular}{|c|c|c|c|c|c|c|c|c|}
\hline \multirow{2}{*}{$\begin{array}{c}\text { IMRT } \\
\text { techniques }\end{array}$} & \multicolumn{4}{|c|}{ Correlations between the joint volume of the bladder and PTV with: } & \multicolumn{4}{|c|}{ Correlations between the joint volume of rectum and PTV with: } \\
\hline & V50 & V60 & Dmean & NTCP & V50 & V60 & Dmean & NTCP \\
\hline $9 \mathrm{~F}$ & 0.93 & 0.96 & 0.86 & 0.84 & 0.89 & 0.93 & 0.81 & 0.78 \\
\hline $7 \mathrm{~F}$ & 0.90 & 0.95 & 0.82 & 0.78 & 0.93 & 0.97 & 0.85 & 0.81 \\
\hline $5 \mathrm{~F}$ & 0.91 & 0.94 & 0.85 & 0.82 & 0.91 & 0.96 & 0.83 & 0.80 \\
\hline \multirow[t]{3}{*}{ BAO } & 0.88 & 0.92 & 0.88 & 0.77 & 0.88 & 0.91 & 0.84 & 0.77 \\
\hline & \multicolumn{4}{|c|}{ Correlations between the distance of the bladder to prostate with: } & \multicolumn{4}{|c|}{ Correlations between the distance of the rectum to prostate with: } \\
\hline & V50 & V60 & Dmean & NTCP & V50 & V60 & Dmean & NTCP \\
\hline $9 \mathrm{~F}$ & -0.89 & -0.92 & -0.91 & -0.83 & -0.85 & -0.89 & -0.81 & -0.77 \\
\hline $7 \mathrm{~F}$ & -0.84 & -0.88 & -0.82 & -0.77 & -0.83 & -0.84 & -0.79 & -0.74 \\
\hline $5 \mathrm{~F}$ & -0.87 & -0.92 & -0.81 & -0.82 & -0.86 & -0.87 & -0.79 & -0.75 \\
\hline BAO & -0.80 & -0.86 & -0.76 & -0.75 & -0.78 & -0.82 & -0.77 & -0.73 \\
\hline
\end{tabular}



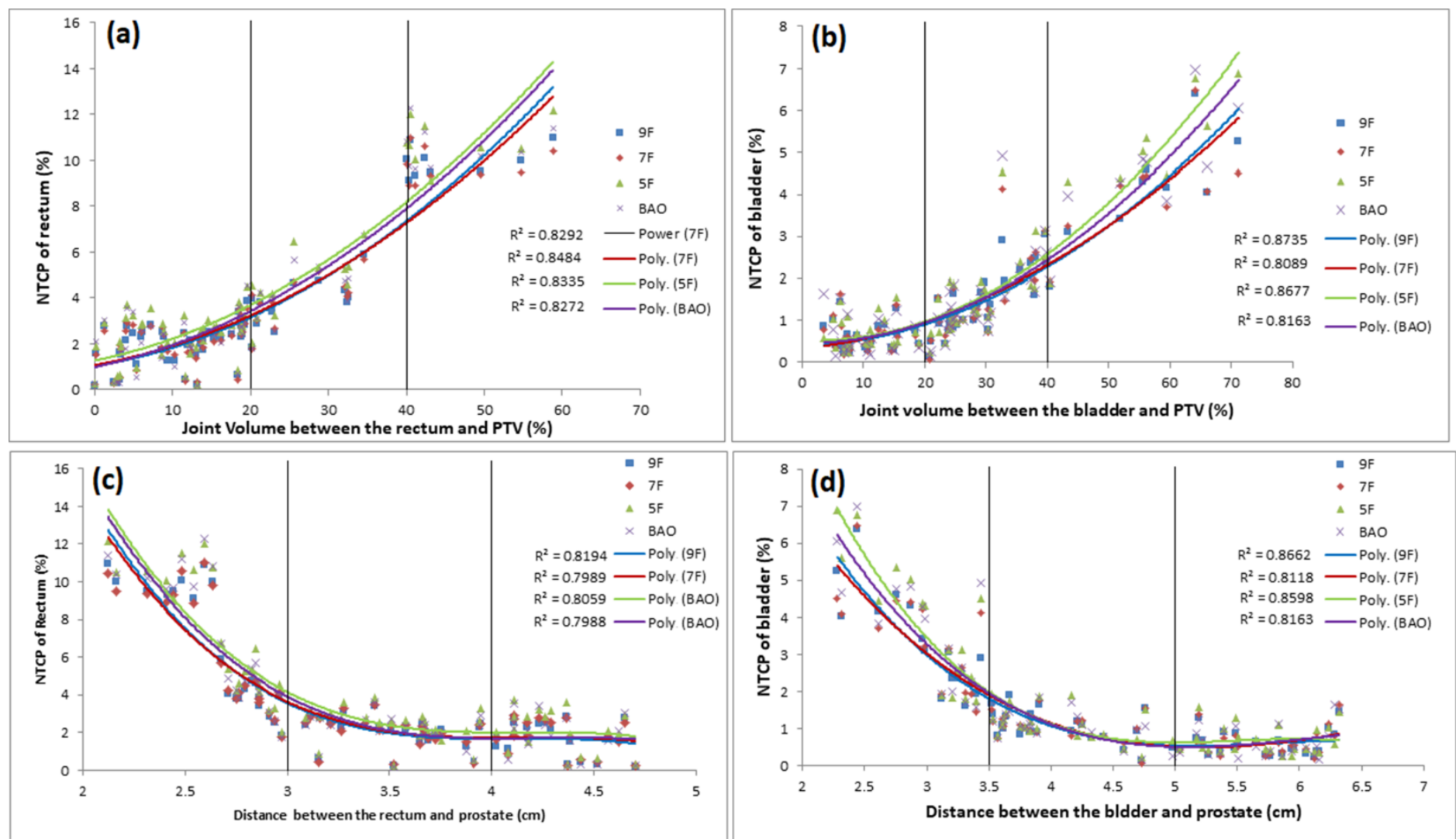

Figure 1. The relationship between the NTCP values and anatomical parameters of OARs for all the patients. (a): The relationship between the NTCP of rectum and joint volumes of rectum with PTV; (b): The relationship between the NTCP of bladder and joint volumes of bladder with PTV; (c): The relationship between the NTCP of rectum and its distance from the prostate centre; (d): The relationship between the NTCP of bladder and its distance from the prostate centre. $* R^{2}$ values for fitting 3-degree polynomial curves represented for relationship between NTCP values and anatomical parameters for each IMRT technique in the figures.
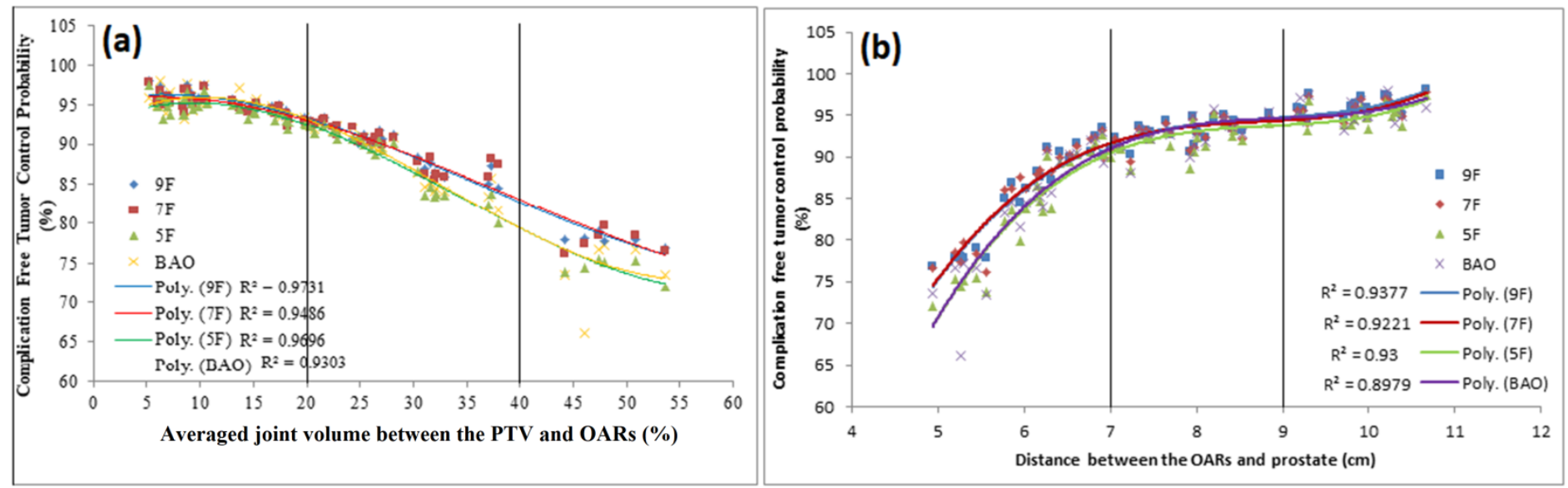

Figure 2. The relationship between the Overall NTCP values and anatomical parameters for all the patients. (a): The relationship between the overall NTCP and average joint volumes of OARs with PTV; (b): The relationship between the overall NTCP and sum of the distances between the prostate and OARs central positions. $* \mathbf{R}^{2}$ values for fitting 3-degree polynomial curves represented for relationship between NTCP values and anatomical parameters for each IMRT technique in the figures. 

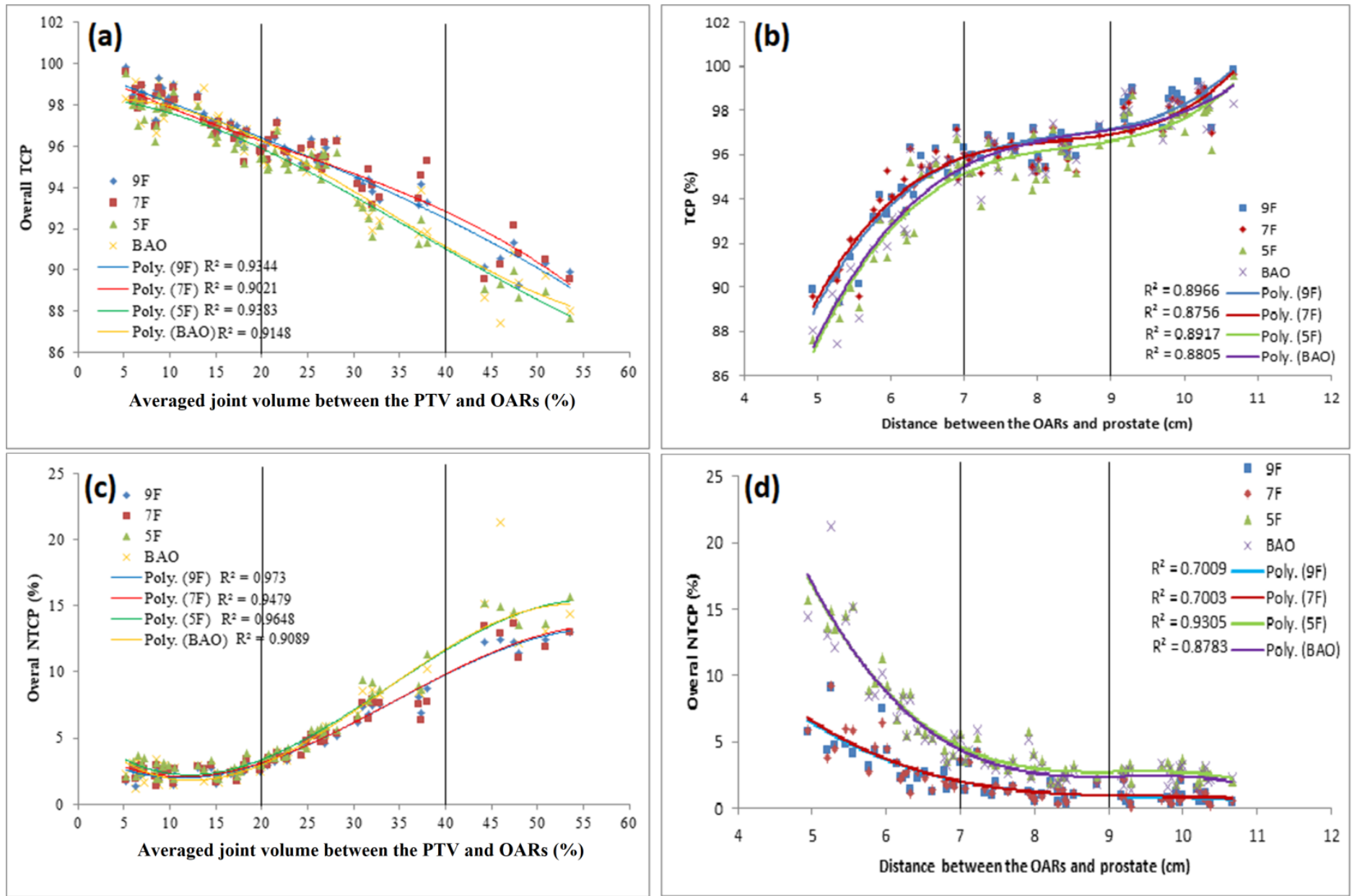

Figure 3. The relationship between the anatomical parameters with TCP and $P_{+}$values in all the patients. (a): The relationship between the TCP and average joint volumes of OARs with PTV; (b): The relationship between the $P_{+}$and averaged joint volumes of OARs with PTV; (c): The relationship between the TCP and sum of the distances between the OARs and prostate central positions; (d): The relationship between the $P_{+}$and sum of the distances between the OARs and prostate central positions. $* \mathbf{R}^{2}$ values for fitting 3-degree polynomial curves represented for each relationship between the anatomical parameters with TCP and $P_{+}$valuesfor each of IMRT technique in the figures.

Figure 3 shows the relationship between the anatomical parameters with TCP and $\mathrm{P}_{+}$. In the patients with average joint volumes ranged from $5 \%$ to $20 \%$ there was no significant differences (P-value>0.07) among various IMRT techniques regarding to their TCP and $\mathrm{P}_{+}$values (Figure 3a and 3b). For the other ranges (20\% to $55 \%$ ), the 9 and 7 fields were found to have significant higher TCP and $\mathrm{P}_{+}$values ( $\mathrm{P}$-value $\left.<0.05\right)$.

Regarding the sum of the distances between the OARs and prostate central positions, statistical analysis showed that in the range of $5 \mathrm{~cm}$ to $7 \mathrm{~cm}$, the 9 and 7 fields have significant higher TCP values ( $\mathrm{P}$-value $<0.04)$. However, the 5 fields and BAO techniques had the same results at this range. In the range of $7 \mathrm{~cm}$ to $9 \mathrm{~cm}$, the 5 fields IMRT technique had significant lower values compared with other techniques ( $\mathrm{P}$-value $<0.05)$. All of the IMRT techniques indicated no significant differences (P-value $>0.14$ ) for the patients with OARs to prostate distances higher than $9 \mathrm{~cm}$ (Figure 3c). The $\mathrm{P}_{+}$showed similar behavior as the TCP with the variation of the OARs to prostate distances, except that there was no statistically differences (Pvalue>0.06) between the IMRT techniques for the patients with distances higher than $7 \mathrm{~cm}$ (Figure 3d).

\section{Discussion}

We evaluated and compared the radiobiological results of four various IMRT techniques in different ranges of anatomical parameters. In the previous studies [4-10], comparison of IMRT radiotherapy techniques with other methods have been investigated from the perspective of radiobiological effects. Nevertheless, different IMRT techniques have not been conclusively compared. There were also several studies for investigating beam orientation and numbers effects in IMRT [11-13]. However, they usually did not compare different available or common techniques to show which technique have more satisfying results, maybe because of different patients anatomical specifications.

We evaluated various anatomical parameters of prostate cancer patients. We did not found any high correlations between these anatomical parameters and radiobiological/ dosimetric parameters, except two of them including distances and joint volumes between the target tissue and OARs. These two parameters included the target and OARs specifications together in one parameter. The anatomical parameters of one of the target or OARs have not the ability to affect the IMRT 
dosimetric/radiobiological results. For example, in the patients with smaller OARs volumes, it is possible that the PTV has large volume, so the OARs will have higher doses/NTCPs due to higher joint volumes. It is also possible that the PTV has small volume, therefore dosimetric and radiobiological parameters of OARs will be lower. Other studies about the effect of anatomical parameters in IMRT, mentioned these two parameters $[14,15]$, but they did not compare IMRT techniques with each other and they did not report the appropriate IMRT techniques regarding to these anatomical parameters.

We showed that in small joint volume fractions and large distances between the OARs and target tissue, there were no statistically differences between various IMRT techniques. Actually, we found critical points for the assessed anatomical parameters. 3 and $3.5 \mathrm{~cm}$ are the critical distances between the center of prostate with rectum and bladder respectively, that in patients with lower distances than these critical values, 9 and 7 fields techniques indicated statistically lower NTCP values. Furthermore, these two IMRT techniques ( 9 and 7 fields) had lower NTCP values in joint volumes higher than the $20 \%$ and $40 \%$ for rectum and bladder respectively. We showed that in distances higher than the $7 \mathrm{~cm}$ between the center of OARs and prostate (sum of distances) there are no any statistical differences in $\mathrm{P}_{+}$parameter. Furthermore, in average joint volumes smaller than the $20 \%$, there were not any differences in TCP and $\mathrm{P}_{+}$between various IMRT techniques. In smaller distances or higher joint volumes, usually the 9 and 7 fields showed better results in OARs and also in target tissue. It was interesting that the 7 fields technique had good results as the 9 fields one. Therefore, it is a good idea to use the 7 fields technique instead of the 9 fields because of lower positioning errors and irradiation times.

The priorities of OAR sparing and target tissue irradiation are important factors in IMRT planning. Regarding to our results, if for one, the OARs sparing has higher priority compared to tumor control, the NTCP-anatomical parameters curves could be useful. With knowing the anatomical parameters, one can find the NTCP differences between the IMRT techniques. In another hand, if tumor control has higher priority, the TCP-anatomical parameters curves and differences between the IMRT techniques, could be useful. The $\mathrm{P}_{+}$versus anatomical parameters curves will be appropriate when the OARs sparing and tumor control had the same priorities.

Regarding to Pearson correlation coefficients resulted between the OARs anatomical and dosimetric/radiobiological parameters, radiobiological findings approved the dosimetric results. However, the correlation coefficients between the anatomical-radiobiological parameters, usually had lower values (Table 2). We also reported the polynomial (degree 3) $\mathrm{R}^{2}$ coefficients for data in the radiobiological-anatomical parameter curves. The exponential and linear coefficients were also evaluated (although their results were not presented), but polynomial curves showed better fittings.
With considering our results, one can find that when there is a significant difference between the IMRT techniques regarding to patient anatomical parameters. Furthermore, the effect of changing the IMRT techniques in OARs and target tissue radiobiological results could be found in our results. These results would be very helpful for choosing appropriate technique and it could be useful for constructing future automatic prostate IMRT planning algorithms.

It should be noted that the femur heads NTCP values were very low, therefore we did not report the results of this structure assuring no remarkable bone damage in all of the techniques. In addition, previous results support our claim $[6,8]$.

We used OARs and target tissue dose limitations based on the previous study by Pollak et al. [17], for IMRT inverse planning procedure. We used this limitations because they are stricter in comparison with other protocols [20,21], and regarding to the previous study by the Mavroidis et al. [4], stricter limitations leaded to better dosimetric and radiobiological results regarding to radiotherapy goals.

\section{Conclusion}

We found high correlations between the dosimetric/ radiobiological parameters of OARs with the joint volumes and with the distances between the OARs and target tissue in all the techniques. In all of the IMRT techniques, the TCP and $\mathrm{P}_{+}$ values were decreased with increasing the joint volume and decreasing with the distances between the OARs and target tissue (as poly-nominal functions). The NTCP values were increased with increasing the joint volumes and decreasing the distances as 3-degree poly-nominal functions.

3 and $3.5 \mathrm{~cm}$ are the critical distances between the central positions of prostate with rectum and bladder respectively, that in patients with lower distances than these critical values, 9 and 7 fields techniques indicated statistically lower NTCP values. Furthermore, these two IMRT techniques (9 and 7 fields) had lower NTCP values in joint volumes higher than the $20 \%$ and $40 \%$ for the rectum and bladder respectively. The TCP and $\mathrm{P}_{+}$ of various IMRT techniques showed no statistical differences in low percentage of joint volumes $(<20 \%)$ and high distances $(>7 \mathrm{~cm})$. Based on our results, it would be possible to compare radiobiological effects of various common IMRT techniques and choose the best one regarding to patients anatomical parameters derived from the CT scans.

\section{Acknowledgements}

The authors express their sincere appreciation to the Radiotherapy and Oncology Department of Shohaday-e-Tajrish Hospital for their financial help and technical assistance. 


\section{References}

[1] Center MM, Jemal A, Lortet-Tieulent J, et al. International variation in prostate cancer incidence and mortality rates. Eur Urol. 2012;61(6):1079-1092.

[2] Khan FM, Gerbi BJ. Treatment planning in radiation oncology. Wolters Kluwer Health/Lippincott Williams \& Wilkins; 2012.

[3] Pan HY, Jiang J, Hoffman KE, et al. Comparative toxicities and cost of intensity-modulated radiotherapy, proton radiation, and stereotactic body radiotherapy among younger men with prostate cancer. J Clin Oncol. 2018;36(18):1823-1830.

[4] Mavroidis P, Komisopoulos G, Buckey C, et al. Radiobiological evaluation of prostate cancer IMRT and conformal-RT plans using different treatment protocols. Phys Med. 2017;40:33-41.

[5] Luxton G, Hancock SL, Boyer AL. Dosimetry and radiobiologic model comparison of IMRT and 3D conformal radiotherapy in treatment of carcinoma of the prostate. Int J Radiat Oncol Biol Phys. 2004;59(1):267-284.

[6] Deb P, Fielding AL. Radiobiological model comparison of 3D conformal radiotherapy and IMRT plans for the treatment of prostate cancer. Australas Phys Eng Sci Med. 2009;32(2):51-61.

[7] Mesbahi A, Rasouli N, Mohammadzadeh M, et al . Comparison of Radiobiological Models for Radiation Therapy Plans of Prostate Cancer: Three-dimensional Conformal versus Intensity Modulated Radiation Therapy. J Biomed Phys Eng. 2018;8(3):1-12.

[8] Khan MI, Jiang R, Kiciak A, et al. Dosimetric and radiobiological characterizations of prostate intensity-modulated radiotherapy and volumetric-modulated arc therapy: A single-institution review of ninety cases. J Med Phys. 2016;41(3):162-168.

[9] Chow J, Jiang R, Kiciak A. Dose-volume consistency and radiobiological characterization between prostate IMRT and VMAT plans. Int J Cancer Ther Oncol. 2016;4(4).

[10] Chow JC, Jiang R, Kiciak A, Markel D. Dosimetric comparison between the prostate intensity-modulated radiotherapy (IMRT) and volumetric-modulated arc therapy (VMAT) plans using the planning target volume (PTV) dose-volume factor. J Radiother Pract. 2016;15(3):263-268.

[11] Schreibmann E, Xing L. Feasibility study of beam orientation class-solutions for prostate IMRT. Med Phys. 2004;31(10):2863-2870.

[12] Schreibmann E, Lahanas M, Xing L, et al. Multiobjective evolutionary optimization of the number of beams, their orientations and weights for intensity-modulated radiation therapy. Phys Med Biol. 2004;49(5):747-770.

[13] Yang R, Dai J, Yang Y, et al. Beam orientation optimization for intensity-modulated radiation therapy using mixed integer programming. Phys Med Biol. 2006;51(15):3653-3666.

[14] Yuan L, Ge Y, Lee WR, et al. Quantitative analysis of the factors which affect the interpatient organ-at-risk dose sparing variation in IMRT plans. Med Phys. 2012;39(11):6868-6878.

[15] Wu B, Ricchetti F, Sanguineti G, et al. Patient geometry-driven information retrieval for IMRT treatment plan quality control. Med Phys. 2009;36(12):5497-5505.

[16] Källman P, Agren A, Brahme A. Tumour and normal tissue responses to fractionated non-uniform dose delivery. Int J Radiat Biol. 1992;62(2):249-262.

[17] Pollack A, Walker G, Horwitz EM, et al. Randomized trial of hypofractionated external-beam radiotherapy for prostate cancer. J Clin Oncol. 2013;31(31):3860-3868.

[18] Sanchez-Nieto B, Nahum AE. BIOPLAN: software for the biological evaluation of radiotherapy treatment plans. Med Dosim. 2000;25(2):71-76.

[19] Hodapp N. The ICRU Report 83: prescribing, recording and reporting photon-beam intensity-modulated radiation therapy (IMRT). Strahlenther Onkol. 2012;188(1):97-99.

[20] Lee WR, Amin MB, Bruner DW, et al. A phase III randomized study of hypofractionated 3D-CRT/IMRT versus conventionally fractionated 3D-CRT/IMRT in patients with favorable-risk prostate cancer. Clinical Trial NCT00331773.

[21] Pollack A, Hanlon AL, Horwitz EM, et al. Dosimetry and preliminary acute toxicity in the first 100 men treated for prostate cancer on a randomized hypofractionation dose escalation trial. Int J Radiat Oncol Biol Phys. 2006;64(2):518-526. 